Research Article

\title{
Evaluation of medication adherence patterns amongst anti-hypertensive drug users in a tertiary care hospital in north India
}

\author{
Mahesh Chander Gupta ${ }^{1}$, Ankita Bhattacharjee ${ }^{1 *}$, Harpreet Singh ${ }^{2}$
}

\begin{abstract}
${ }^{1}$ Department of Pharmacology, Pt. B. D. Sharma Post Graduate Institute of Medical Sciences, Rohtak, India

${ }^{2}$ Department of Medicine, Pt. B. D. Sharma Post Graduate Institute of Medical Sciences, Rohtak, India
\end{abstract}

Received: 06 August 2016

Accepted: 10 September 2016

\section{*Correspondence to:}

Dr. Ankita Bhattacharjee, Email: ankitabhattacharjee49@ gmail.com

Copyright: (C) the author(s), publisher and licensee Medip Academy. This is an openaccess article distributed under the terms of the Creative Commons Attribution NonCommercial License, which permits unrestricted noncommercial use, distribution, and reproduction in any medium, provided the original work is properly cited.

\begin{abstract}
Background: Non-adherence to prescribed medications is a major barrier in management of chronic diseases like hypertension, which can lead to treatment failure and can increase morbidity, mortality and health care costs. The objective of this study was to assess the level of adherence of hypertensive patients to their anti-hypertensive medications and various factors influencing the adherence among these patients.

Methods: A cross sectional, observational, questionnaire based study was carried out amongst 70 patients diagnosed with hypertension. Adherence was measured using Morisky medication adherence score questionnaire (MMAS-8). Co-relation of various factors like age, sex, education level, number of drugs taken, presence of concomitant diseases etc. were assessed. Univariate and multivariate analysis was done to determine the factors associated with adherence.

Results: The mean age of 70 patients (35 male, 35 female) were $52.29 \pm 11.39$ years. Using MMAS-8, high, medium and low adherence was seen in $58.6 \%$, $20.0 \%$ and $21.4 \%$ patients respectively. Middle age group patients were more adherent $(60.8 \%)$ than younger age group and elderly $(37.5 \%)$ (p-value $<0.05)$. Adherence rates were comparable in men and women. There was a positive correlation of adherence with education level, knowledge of BP goals and presence of diabetes. It showed negative correlation with chronicity of hypertension and number of drugs taken.

Conclusions: Medication adherence rate was found to be low in the present study. The level of adherence to treatment among the participants can be achieved through better health promotion and education strategies.
\end{abstract}

Keywords: Medication, Adherence, Hypertension, MMAS-8

\section{INTRODUCTION}

Hypertension is a silent killer disease in both the developed and developing nations of the world. Increased blood pressure is a leading risk factor for premature death, stroke and heart disease worldwide. ${ }^{1}$ According to WHO, one in three adults worldwide, has raised blood pressure which is one of the major risk factor for coronary heart disease and stroke. WHO data indicate that by 2025 the global burden of hypertension will increase by $60 \%$ to be 1.56 billion individuals worldwide and higher in the developed nations. ${ }^{2}$ In India, review of epidemiological studies suggests that the prevalence of hypertension has increased in both urban and rural subjects and presently is $25 \%$ in urban adults and $10-15 \%$ among rural adults. ${ }^{3}$ This indicates that adherence to antihypertensive medication is not good in our country, which has made hypertension an increasingly important medical and public health issue. The World Health Organization (WHO) has estimated that about $62 \%$ of cerebro-vascular disease and $49 \%$ of ischemic heart disease burden worldwide are attributable to suboptimal blood pressure levels. High blood pressure is estimated to cause 7.1 million deaths annually, accounting for $13 \%$ of all deaths globally.

According to the World Health Organization (WHO), among patients with chronic illness, about $50 \%$ do not 
take medications as prescribed. ${ }^{3}$ Medication nonadherence is a major public health problem that has been called an "invisible epidemic." ${ }^{5}$ Non-adherence to the medical regimens is a major concern in the management of patients with chronic illness like hypertension and is a major cause for treatment failure. ${ }^{3,6}$

It is an important and often unrecognized risk factor that contributes to a reduced control of blood pressure. Studies have shown that $50 \%$ of individuals discontinue antihypertensive medications within 6 to 12 months of their initiation. ${ }^{7}$

Adherence with medication usage is defined as the proportion of prescribed doses of medication actually taken by a patient over a specified period of time or in simpler terms extent to which individuals follow the instructions given for prescribed treatment. ${ }^{8}$ Nonadherence to prescribed medications cause treatment failure and lead to unnecessary visits to physician, prescription changes, frequent re-hospitalizations and increase morbidity, mortality and health care costs. Therapeutic non-adherence occurs when an individual's health-seeking or maintenance behaviour lacks congruence with the recommendations as prescribed by a healthcare provider. ${ }^{9,10}$

The term "adherence", implies an active role in collaboration with a prescriber, and "non-adherence" encompasses the diverse reasons for patients not following a treatment recommendation. ${ }^{11}$ Intentional and unintentional patterns of non-adherence have been recognized. Intentional non-adherence is an active process whereby the patient chooses to deviate from the treatment regimen. This may be a rational decision process in which the individual weighs the risk and benefits of treatment against any adverse effects. Unintentional non-adherence is a passive process whereby the patient may be careless or forgetful about adhering to the treatment regimen. ${ }^{12,13}$

Patients are generally considered adherent to their medication if their medication adherence percentage, defined as the number of pills absent in a given time period ("X") divided by the number of pills prescribed by the physician in that same time period, is greater than $80 \%$. $^{8,14}$

Measurement of medication adherence is challenging because adherence is an individual patient behaviour. The following are some of the approaches that have been used: (a) subjective measurements obtained by asking patients, family members, caregivers, and physicians about the patient's medication use; (b) objective measurements obtained by counting pills, examining pharmacy refill records, or using electronic medication event monitoring systems; and (c) biochemical measurements obtained by adding a nontoxic marker to the medication and detecting its presence in blood or urine or measurement of serum drug levels. A multi- method approach that combines feasible self-reporting and reasonable objective measures is the current state of the-art in measurement of adherence behavior. ${ }^{14}$ Adherence to antihypertensive therapy can be approximated by measuring blood pressure (BP) control.

Non-adherence to long-term medications in chronic diseases is a worldwide problem. Evidence from a number of studies suggests that adherence rates varied from around $50-88 \%$ for patients treated for hypertension. $^{15-18}$

According to the World Health Organization (WHO), in developed countries, non-adherence of patients with chronic diseases is around $50 \% .^{3}$ The magnitude and impact of poor adherence in developing countries is assumed to be even higher given the paucity of health resources and inequities in access to health care. Vast amount of literature is available on medication adherence pattern of hypertensive subjects. Data is available from study conducted in various developed and developing nations, various clinical/ hospital settings, on urban and rural subjects etc. In one US study, Monane et al found that antihypertensive compliance averaged $49 \%$, and only $23 \%$ of the patients had good compliance levels of $80 \%$ or higher16. A study conducted in Nigeria found $44.7 \%$ were good drug adherers while $55.3 \%$ were not so. ${ }^{17}$ Similar study carried out in Malaysia, showed high adherence rate to be $54 \% .^{19}$ Evidence from studies conducted in India suggest that compliance is affected by various demographic features such as rural/urban settings, assess to heath care, regimen complexity etc. ${ }^{20,21}$

Past research has identified a range of factors that may affect medication adherence. The WHO classifies the factors which affect adherence into 5 categories: patientrelated factors, therapy-related factors, socioeconomic factors, disease-related factors, factors associated with the health care team and system in place. These factors have been explored as important determinants of compliance. In broader terms, these factors fall into the categories of patient-related factors, physician-related factors, and health system/team building-related factors. ${ }^{14}$

Numerous studies indicate that low adherence to antihypertensive drugs affects blood pressure control, rate of complications, and overall health care costs. ${ }^{2,23}$

High adherers (adherence more than 80\%) had a significantly lower risk of cardiovascular events in comparison with low adherers (adherence, 40\%) after an average follow-up duration. ${ }^{24}$

The relationship between health knowledge and compliance was highly supported in some studies. Data in literature suggested that patients who knew about the importance of compliance had a significantly higher compliance rate than did those who had been ignorant of that aspect. ${ }^{18,19}$ This stresses the importance of health education to increase the knowledge of hypertension and 
its sequel to improve patient's non-compliance behaviour.

To improve adherence we have to identify the factors for causing noncompliance, to detect it, and to improve and/or maintain vigilance. No single intervention strategy is superior for improving drug compliance. Sequential barriers exist to drug adherence in hypertension, ie, poor communication, low motivation, complexity of treatment, acute and delayed side effects, limited long-term motivation, and reinforcement of poor habits. ${ }^{12}$ There is no "gold standard" for measuring adherence behavior and the use of a variety of strategies has been reported in the literature. ${ }^{25}$

According to the WHO 2008 estimates, the prevalence of raised $\mathrm{BP}$ in Indians was $32.5 \%(33.2 \%$ in men and $31.7 \%$ in women). However, only about $25.6 \%$ of treated patients had their BP under control. ${ }^{26}$ This high incidence of hypertension and the incidence of complications even among those on treatment due to poor compliance, explain the need for more awareness and with emphasis on compliance.

The purpose of present study was to evaluate the extent of adherence to anti-hypertensive therapy and to identify various factors influencing the adherence among hypertensive.

\section{METHODS}

\section{Study population and design}

This study was performed at Pt. B. D. Sharma Post Graduate Institute of Medical Sciences, Rohtak. Physician diagnosed hypertensive patients with ongoing treatment, from Medicine OPD were included in the study, they were screened with the help of a pre-defined inclusion and exclusion criteria for the study. Inclusion criteria includes patients of either gender aged $\geq 18$ years with confirmed diagnosis of hypertension and taking antihypertensive drugs and willing to participate in the study and ready to give informed consent. Exclusion criteria were newly diagnosed hypertensive patients, pregnancy and lactation, dementia (MMSE<24), subjects who are taking other forms of medication (Ayurvedic/Homeopathic). ${ }^{27}$

The study was cross sectional, observational, questionnaire based study carried out amongst 70 patients diagnosed with hypertension. Demographic and clinical characteristics was recorded of all the eligible patients like age, gender, history of duration of hypertension, socioeconomic status, education level, co-morbidities like diabetes, obesity, dyslipidemia, others were noted. Questions also asked on number and frequency of the drugs and any side effects of drugs as experienced by patient, knowledge of target blood pressure and risk of poor blood pressure control.

\section{Outcome measures}

Adherence was measured using Morisky medication adherence score questionnaire (MMAS-8) ${ }^{28}$ The Morisky medication adherence scale (MMAS) is a generic selfreported, medication-taking behaviour scale which has been validated for use to assess adherence in hypertension. MMAS-8 consists of eight items with a scoring scheme of "Yes" = 0 and "No" = 1 for the first seven items and a 5-point likert response for the last item. The items are summed to give a range of scores from low adherence to high adherence. $^{28,29}$

This questionnaire was administered by the attending clinician at the time other data as stated above were collected. Co-relation of various factors like age, sex, education level, number of drugs taken, presence of concomitant diseases etc. were assessed.

The degree of adherence was determined according to the score resulting from the sum of all the correct answers: high adherence ( 8 points), average adherence ( 6 to $<8$ points) and poor adherence ( $<6$ points). In this study, patients were considered adherent when they had a score equal to eight in the MMAS-8.

\section{Statistical analysis}

The data analysis was carried out using Statistical package for social science (SPSS), version 21. Basic descriptive statistics using mean and standard deviation were presented for numerical data variables such as the number of drugs used and knowledge scores. The categorical data such as age category, gender, education, presence of co-morbidity, duration of disease, number of drugs taken, and knowledge about complications of hypertension were presented as frequency and percentage. The association between compliance and different variables was assessed by chi-square test. pvalue of $<0.05$ was considered as statistically significant.

\section{RESULTS}

\section{Demographics and health status}

A total of 78 patients were screened for this study. Out of these 8 patients were excluded as they did not match the predefined inclusion criteria i.e. 5 patients were newly diagnosed with hypertension, 1 did not give informed consent, 1 was a pregnant female and 1 patient patients was excluded as he was mentally subnormal.

The baseline demographic characteristics are given in Table 1 .

The mean age of the study population was $52.29 \pm 11.39$ years, out of which males and females comprised $50 \%$ each ( 35 males and 35 females). Their ages ranged from 22 to 75 years. A small percentage $8(11.4 \%)$ was below 40 years, the majority, $46(65.7 \%)$ of the subjects were in 
the 40 to 60 year old range, and $16(22.8 \%)$ were above age of 60 years.

Table 1: Demographic and health status of the patients.

\begin{tabular}{|ll|}
\hline Demographic parameters & $\begin{array}{l}\text { Value/number of } \\
\text { patients }\end{array}$ \\
\hline Age & $52.29 \pm 11.39$ years \\
\hline Age range & $22-75$ years \\
\hline$<40$ years & $8(11.4 \%)$ \\
\hline $40-60$ years & $46(65.7 \%)$ \\
\hline$>60$ years & $16(22.8 \%)$ \\
\hline Female: Male & $35(50 \%): 35(50 \%)$ \\
\hline Education & $20(29 \%)$ \\
\hline Elementary & $50(71 \%)$ \\
\hline High school and above & 6 months- 26 years \\
\hline $\begin{array}{l}\text { Diagnosed with hypertension } \\
\text { since }\end{array}$ & $35(50 \%)$ \\
\hline $\begin{array}{l}\text { Newly diagnosed hypertensives } \\
(<5 \text { years) }\end{array}$ & $42(60 \%)$ \\
\hline Concomitant diabetes & \\
\hline
\end{tabular}

Education wise $20(29 \%)$ patients had completed elementary education while $50(71 \%)$ had completed high school or above. Patients included in the study had been diagnosed with hypertension for 6 months to 26 years. Among all the patients sampled, 35 patients or $50 \%$, were relatively new hypertensives, having been diagnosed with hypertension within only 5 years. Out of these, $25 \%$ had been hypertensive for 3 years or fewer. Only 11 patients $(15.8 \%)$ had been hypertensive for more than 10 years. Of the 70 hypertensive patients sampled, $42(60 \%)$ patients had concomitant diabetes.

Table 2: Frequency distribution of adherers and nonadherers.

\begin{tabular}{|lllll|}
\hline $\begin{array}{l}\text { Adherence } \\
\text { score } \\
\text { MMacc.to }\end{array}$ & $\begin{array}{l}\text { Adherence } \\
\text { status }\end{array}$ & Frequency & Percentage \\
\hline 8 & High & Adherers & 41 & $58.6 \%$ \\
\hline 7 & Medium & Non-adherers & 9 & $12.9 \%$ \\
\hline 6 & Medium & Non-adherers & 5 & $7.1 \%$ \\
\hline 5 & Low & Non-adherers & 9 & $12.9 \%$ \\
\hline 4 & Low & Non-adherers & 6 & $8.5 \%$ \\
\hline Total & & & $\mathbf{7 0}$ & $\mathbf{1 0 0} \%$ \\
\hline
\end{tabular}

\section{Adherence scores}

Using MMAS-8, adherence scores obtained in the present study ranged from 4 to 8 . From the scores obtained, the sampled patients were categorized either as adherers or non-adherers. The frequency distribution is shown in Table 2. Score of 8 was taken as high adherence, score of 6 and 7 as medium adherence, score of 5 and below as low adherence. By this scoring category, high adherence was seen in $58.6 \%$ respondents. Out of remaining $20 \%$ were medium adherers and $21.4 \%$ were low adherers.



Figure 1: Adherence level.

Adherence rates were comparable in men and women. Middle age group patients were more adherent (67.4\%) than younger age group and elderly $(41.7 \%)$. There was a positive correlation of adherence with education level, knowledge of BP goal. It showed negative correlation with chronicity of hypertension, number of drugs taken and presence of any co-morbidity.

\section{DISCUSSION}

The results of the study showed that in the centre conducted; only $58.6 \%$ patients were highly adherent to their medication regimen. Reviews of literature from various similar studies in different countries conclude that medication adherence is affected by various parameters which vary widely from region to region. In the current study's grading, unintentionally missing a dose would also label the patient as not being non-adherent. This might account for the relatively huge figure of nonadherents. On most occasions, missing a dose was mainly due to patients' forgetfulness. This could be improved by advising them to correspond medication taking with certain activities eg. after meals or to set reminders.

The effects of various demographic and other features on adherence were assessed. Among the demographic variables studied, males were found to be slightly more adherent to their medication regimen, but this difference was not statistically significant. Among the different age categories, the middle aged group (40-60 years) were found to be better compliers $(67.4 \%)$ than the young $(<40$ years) and the elderly (>60 years) in whom only $62.5 \%$ and $31.2 \%$ respectively were found to be adherent. The increase in adherence with age may be due to greater concern regarding health with advancing age. The young patients tend to keep busy and have a lack of concern about their health. Similar results were observed from a study among diabetic patients in Malaysia, which showed that non-compliers were largely younger patients. ${ }^{19}$ This mandates the need for targeting the young age group and elderly to improve their compliance so as to prevent future catastrophes.

Also it was seen that with chronicity of hypertension and increasing number of drugs taken, the rate of compliance decreased substantially. Good health awareness and knowledge of high blood pressure, as well as of the 
medications being taken, have been shown to be associated with good adherence to medication regimes in several studies. ${ }^{18,19}$ In the present study, there was a positive correlation of adherence with education level, knowledge of BP goal. Patients who knew about the importance of compliance had a significantly higher compliance rate $(72.7 \%)$ than did those who had been ignorant of that aspect. This stresses the importance of health education in improving patient's adherence.

Table 3: Association between adherence and other variables.

\begin{tabular}{|c|c|c|c|c|c|c|}
\hline Parameters & No. of patients & Adherers & Percentage & Non-adherers & Percentage & p- value \\
\hline \multicolumn{6}{|l|}{ Age category } & \multirow{4}{*}{0.080} \\
\hline Young & 8 & 5 & $62.5 \%$ & 3 & $37.5 \%$ & \\
\hline Middle & 46 & 31 & $67.4 \%$ & 15 & $32.6 \%$ & \\
\hline Elderly & 16 & 5 & $31.2 \%$ & 11 & $68.8 \%$ & \\
\hline \multicolumn{6}{|l|}{ Gender } & \multirow{3}{*}{0.242} \\
\hline Male & 35 & 21 & $60.0 \%$ & 14 & $40.0 \%$ & \\
\hline Female & 35 & 20 & $57.1 \%$ & 15 & $42.9 \%$ & \\
\hline \multicolumn{6}{|l|}{ Education } & \multirow{3}{*}{ 0.008* } \\
\hline Elementary & 20 & 9 & $45.0 \%$ & 11 & $55.0 \%$ & \\
\hline High school and above & 50 & 32 & $64.0 \%$ & 18 & $36.0 \%$ & \\
\hline \multicolumn{6}{|l|}{ Presence of diabetes } & \multirow{3}{*}{0.129} \\
\hline Yes & 42 & 22 & $52.4 \%$ & 20 & $47.6 \%$ & \\
\hline No & 28 & 19 & $67.8 \%$ & 9 & $32.2 \%$ & \\
\hline \multicolumn{6}{|c|}{ Duration of hypertension (years) } & \multirow{4}{*}{0.168} \\
\hline$\leq 5$ & 35 & 24 & $68.6 \%$ & 11 & $31.4 \%$ & \\
\hline $6-10$ & 24 & 13 & $54.2 \%$ & 11 & $45.8 \%$ & \\
\hline$>10$ & 11 & 4 & $36.4 \%$ & 7 & $63.6 \%$ & \\
\hline \multicolumn{6}{|c|}{ Knowledge about complication of hypertension } & \multirow{3}{*}{$0.001 *$} \\
\hline Yes & 55 & 40 & $72.7 \%$ & 15 & $27.3 \%$ & \\
\hline No & 15 & 1 & $6.7 \%$ & 14 & $93.3 \%$ & \\
\hline \multicolumn{6}{|l|}{ Number of drugs taken } & \multirow{4}{*}{0.086} \\
\hline 1 & 41 & 26 & $63.4 \%$ & 15 & $36.6 \%$ & \\
\hline 2 & 19 & 11 & $57.9 \%$ & 8 & $42.1 \%$ & \\
\hline$>2$ & 10 & 4 & $40.0 \%$ & 6 & $60.0 \%$ & \\
\hline
\end{tabular}

Majority of the non-compliant patients in the present study reported forgetfulness and absence of symptoms of hypertension as reasons for their non-compliance. Other reasons reported were presence of drugs side effects and drugs out of supply. These findings are similar to those of another study where almost half of the subjects reported forgetfulness as the major factor for non-compliance. ${ }^{18,30}$ This stresses the importance of health education to improve patients non-compliant behaviour. Patients should be made aware of the consequences of uncontrolled blood pressure and ensuing futility of treatment. Such behavioural modification will save unnecessary hospital visits and frequent change of regimen will incur heavy financial burden for the patient and also prevent future morbidity and mortality. It was also evident from the study that those patients with higher adherence scores had better control of their blood pressure as compared to those with lesser adherence. Efforts should always be made to identify the reasons for nonadherence and the steps to be taken to improve it, through better communication between health care providers and patients.

\section{CONCLUSION}

The medication adherence rate among hypertensive patients conducted at a tertiary care hospital in north India was found out to be $58.6 \%$. Amongst the factors found to negatively affect adherence were longer duration of disease, larger number of drugs and presence of concomitant diabetes. Whereas with higher education and knowledge regarding complications of uncontrolled blood pressure, adherence rate increased substantially. Therefore it is mandated that healthcare providers identify those factors which affect compliance and work collectively to foster better adherence behaviour amongst patients. Better health promotion and education strategies and intervention programmes should be adopted for long term control of blood pressure. 
Funding: No funding sources

Conflict of interest: None declared

Ethical approval: The study was approved by the Institutional Ethics Committee

\section{REFERENCES}

1. WHO. World Health Organization, World Health Statistics 2012. Geneva: World Health Organization; 2012.

2. Kearney PM, Whelton M, Reynolds K, Muntner P, Whelton PK, He J. Global burden of hypertension: analysis of worldwide data. Lancet. 2005;365:21723.

3. Sabaté E, Editor. Adherence to long-term therapies: evidence for action. Geneva: World Health Organization; 2003.

4. WHO. World Health Organization, Global Health Observatory (GHO) data. WHO; 2015.

5. Optimizing adherence to pharmaceutical care plans. gloria nichols-English, Sylvie Poirier. J Am Pharm Assoc. 2000;40(4).

6. Blandford L, Dans PE, Ober JD, Wheelock C. Analyzing variations in medication compliance related to individual drug, drug class, and prescribing physician. J Managed Care Pharm. 1999;5(1):47-5.

7. Chobanian AV. Impact of nonadherence to antihypertensive therapy. Circulation. 2009;120:1558-60.

8. Osterberg L, Blaschke T. Adherence to medication. N Engl J Med. 2005;353:487-97.

9. Jin J, Sklar GE, Sen Oh VM, Li SC. Factors affecting therapeutic compliance: a review from the patient's perspective. Ther Clin R Managem. 2008;4(1):26986.

10. DiMatteo MR. Variations in patients' adherence to medical recommendations: a quantitative review of 50 years of research. Med Care. 2004;42(3):200-9.

11. Kalogianni A. Factors affect in patient adherence to medication regimen. Health Sci J. 2011;5(3).

12. Krzesinski JM, Leeman M. Practical issues in medication compliance in hypertensive patients. Res Rep Clin Cardiol. 2011;2:63-70.

13. Lowry KP, Dudley TK, Oddone EZ, Bosworth HB. Intentional and unintentional non-adherence to antihypertensive medication. Ann Pharmacother. 2005;39:1198-203.

14. Brown MT, Bussell JK. Medication adherence: WHO Cares? Mayo Clin Proc. 2011;86(4):304-14.

15. Ho PM, Bryson CL, Rumsfeld JS. Medication adherence: its importance in cardiovascular outcomes. Circulation. 2009;119:3028-35.

16. Compliance with antihypertensive therapy among elderly medicaid enrollees: the roles of age, gender, and race. Monane M, Bohn RL, Gurwitz JH, Glynn RJ, Levin R. Avorn J Am J Public Health. 1996;86(12):1805-8.
17. Kabir M, Iliyasu Z, Abubakar IS, Jibril M. Compliance to medication among hyper tensive patients. J Com Med Prim Healt Car. 2004;16(1):1620.

18. Al-Mehza AM, Al-Muhailije FA, Khalfan MM, AlYahya AA. Drug compliance among hypertensive patients; an area based study. Eur J Gen Med. 2009;6(1):6-10.

19. Ramli A, Ahmad NS, Paraidathathu T. Medication adherence among hypertensive patients of primary health clinics in Malaysia. Patie Pref Adher. 2012;6:613-22.

20. Susan R, Anu K, Achu T, Soumya G, Vijayakumar $\mathrm{K}$, Anish TS. Antihypertensive drug compliance across clinic and community settings, in Thiruvananthapuram, South India. Health Sciences. 2012;1(3):JS002A.

21. Kale S, Patil A, Mandlecha RH. Compliance and adverse drug effects of antihypertensives in rural India. J Clin Diagnos Res. 2011;5(4):775-9.

22. Ho PM, Magid DJ, Shetterly SM, Olson KL, Peterson PN, Masoudi FA, et al. Importance of therapy intensification and medication non-adherence for blood pressure control in patients with coronary disease. Arch Intern Med. 2008;168:271-6.

23. Burnier M. Medication adherence and persistence as the cornerstone of effective antihypertensive therapy. Am J Hypertens. 2006;19:1190-6.

24. Mazzaglia G, Ambrosioni E, Alacqua M, Filippi A, Sessa E, Immordino $\mathrm{V}$, et al. Adherence to antihypertensive medications and cardiovascular morbidity among newly diagnosed hypertensive patients. Circulation. 2009;120:1598-605.

25. Mallion JM, Schmitt D. Patient compliance in the treatment of arterial hypertension. European Soci Hypert Scienti Newslet. 2001;2:7.

26. Anchalaa R, Kannuri NK, Pant H, Khan H, Franco $\mathrm{OH}$, Angelantonio E, et al. Hypertension in India: a systematic review and meta-analysis of prevalence, awareness, and control of hypertension. J Hypertens. 2014;32(6):1170-7.

27. Folstein MF, Folstein SE, McHugh PR. Mini mental state. A practical method for grading the cognitive state of the patients for the clinician. J Psychiatr Res. 1975;12(3):189-98.

28. Morisky DE, Green LW, Levine DM. Concurrent and predictive validity of a self-reported measure of medication adherence. Med Care. 1986;24:67-74.

29. Morisky DE, Ang A, Krousel-Wood M, Ward HJ. Predictive validity of a medication adherence measure in an outpatient setting. J Clin Hypertens. 2008;10(5):348-54.

30. Khalil SA, Elzubier AG. Drug compliance among hypertensive patients in Tabuk, Saudi Arabia. J Hypert. 1997;15(5):561-5.

Cite this article as: Gupta MC, Bhattacharjee A, Singh H. Evaluation of medication adherence patterns amongst anti-hypertensive drug users in a tertiary care hospital in north India. Int J Basic Clin Pharmacol 2016;5:2261-6. 